\title{
Acute lymphocytic leukemia mimicking spondyloarthritis in an adolescent: A case report and review of the literature
}

\author{
DANYI XU, GUANHUA XU, LIQIN XU, HENG CAO, BEI XU, WEIQIAN CHEN, \\ CHUANYIN SUN, LIHUAN YUE and JIN LIN \\ Department of Rheumatology, The First Affiliated Hospital, School of Medicine, \\ Zhejiang University, Hangzhou, Zhejiang 310003, P.R. China
}

Received December 1, 2014; Accepted November 19, 2015

DOI: $10.3892 / \mathrm{ol} .2015 .4026$

\begin{abstract}
The present study describes the case of an 18-year-old adolescent male exhibiting acute lymphocytic leukemia (ALL), complicated by the onset of the symptom of sacroiliitis mimicking spondyloarthritis. Atypical features including an enlarged spleen, poor effects of non-steroidal anti-inflammatory drug therapy, low levels of hemoglobin, a low platelet count, a low neutrophil count and increased levels of monocytes, indicated the possibility of hematological malignancy. Bone marrow examination confirmed the diagnosis of ALL. The patient received chemotherapy and the symptoms were dramatically relieved. To the best of our knowledge, the current study reports the second published case of a patient with ALL presenting with sacroiliitis. Sacroiliitis as an onset manifestation of ALL may result in misdiagnosis, therefore, a differential diagnosis is essential when atypical features are present.
\end{abstract}

\section{Introduction}

Paraneoplastic syndromes are the manifestation of an underlying malignancy, which may be hidden, and their pathogenesis is complicated and largely remains to be elucidated. Manifestations of rheumatic disease, particularly arthritis, may be an initial presentation of a paraneoplastic phenomenon that suggests malignancy. Leukemia cases, particularly acute lymphocytic leukemia (ALL), frequently present with arthritic manifestations in childhood, (1) which may lead to the misdiagnosis of rheumatic disease. The peak incidence of ALL is in childhood, arthritic manifestations can occur in $15-30 \%$ of ALL cases especially in children at disease onset, when peripheral blood changes are subtle or even absent (2). ALL

Correspondence to: Ms. Jin Lin, Department of Rheumatology, The First Affiliated Hospital, School of Medicine, Zhejiang University, 79 Qingchun Road, Hangzhou, Zhejiang 310003, P.R. China

E-mail: linjinzju@163.com

Key words: acute lymphocytic leukemia, paraneoplastic syndrome, sacroiliitis, spondyloarthritis frequently affects peripheral joints, while axial spine involvement is rarely observed. Sacroiliitis is a common manifestation of spondyloarthritis, which can be clarified by computer tomography scan of sacroiliac joint (1). Sacroiliitis as an initial manifestation of leukemia mimicking spondyloarthritis is rare; only one similar case was reported in published literature before. Clinicians should be aware that spondyloarthritis may be the onset manifestation of ALL when atypical symptoms and laboratory results are observed. Morphological, immunological phenotype and cytochemical examination of bone marrow are important to identify and classify the underlying leukemia. Chemotherapy appears to have positive effects on arthritic symptoms in these patients. The current study presents the case of an 18-year-old adolescent male who presented with sacroiliitis, and who was eventually diagnosed with ALL. The relevant literature concerning leukemic arthritis (LA) is additionally reviewed.

\section{Case report}

An 18-year-old male presented to the Department of Rheumatology, The First Affiliated Hospital (School of Medicine, Zhejiang University, Hangzhou, Zhejiang, China) in January 2012 with pain in the lower back and left knee. The lower back pain had been apparent for 4 months, and persisted day and night without morning stiffness. Resting did not relieve the pain. The symptom was progressive and became gradually unbearable. The patient experienced pain in the left knee without joint swelling 1 month prior to presenting to the hospital. There was no family history of any arthritic disorders or any form of spondyloarthritis. Computed tomography scans of the sacroiliac joints revealed mild bilateral erosions (Fig. 1), and non-steroidal anti-inflammatory drugs (NSAIDs; meloxicam, $7.5 \mathrm{mg}$ twice a day) were administered, however, the patient demonstrated no response.

Physical examination revealed that the patient's body temperature was $38^{\circ} \mathrm{C}$ and purple-red papules were scattered throughout the anterior chest wall. Sternum tenderness was not observed. The spleen was significantly enlarged. No swelling lymph nodes were present. The sacroiliac joints were bilaterally tender on palpation. The left knee was tender without swelling. Lumbar spine motion was not decreased. 
Routine blood examination revealed the following: A hemoglobin level of $105 \mathrm{~g} / 1$ (normal range, 131-172 $\mathrm{g} / \mathrm{l}$ ), a platelet count $99 \times 10^{9} / 1$ (normal range, $100-300 \times 10^{9} / 1$ ), a white blood cell count of $5.1 \times 10^{9} / 1$ (normal range, $4.0-10.0 \times 10^{9} / 1$ ), a neutrophil count of $1.1 \times 10^{9} / 1$ (normal range, 2.0-7.0x $10^{9} / 1$ ), a lymphocyte count of $2.1 \times 10^{9} / 1$ (normal range, $\left.0.8-4.0 \times 10^{9} / 1\right)$ and a monocyte count of $1.86 \times 10^{9} / 1$ (normal range, $0.12-1.0 \times 10^{9} / 1$ ), with $22.1 \%$ neutrophils (normal range, 50.0-70.0\%), 40.2\% lymphocytes (normal range, 20.0-40.0\%) and $36.3 \%$ monocytes (normal range, $3.0-10.0 \%$, indicating a slight decrease in neutrophils, hemoglobin and platelets, but a significant increase in monocytes. Trace levels of urinary protein were observed. The level of serum high-sensitivity C-reactive protein was $39.2 \mathrm{mg} / \mathrm{l}$ (normal range, $0-8.0 \mathrm{mg} / \mathrm{l}$ ), and the erythrocyte sedimentation rate was $20 \mathrm{~mm} / \mathrm{h}$ (normal range, $0-15 \mathrm{~mm} / \mathrm{h}$ ), indicating elevation. Tests for human leukocyte antigen (HLA)-B27, rheumatoid factor, anti-neutrophil cytoplasmic antibody and antinuclear antibody were negative. Abnormally decreased levels of immunoglobulin (Ig)A (35.7 mg/dl; normal range, $90-450 \mathrm{mg} / \mathrm{dl}$ ), IgG $(649.0 \mathrm{mg} / \mathrm{dl}$; normal range, $800-1,800 \mathrm{mg} / \mathrm{dl})$ and $\mathrm{IgM}$ (33.2 mg/dl; normal range, $60-280 \mathrm{mg} / \mathrm{dl}$ ) revealed hypoglobulinemia. An abdominal Doppler ultrasound revealed significant splenomegaly and kidney swelling with manifestations of nephropathy. Abnormal cells were detected by microscopic examination of peripheral blood smears. Bone marrow aspiration was performed, revealing marked lymphocytes, including many primitive lymphocytes and prolymphocytes $(86 \%$; Fig. 2). Immunophenotypic analysis revealed that the blasts were positive for cluster of differentiation (CD)19, CD22, cytoplasmic IgM and HLA-DR, and pre-B-cell ALL was indicated. The manifestation of sacroiliitis mimicking spondyloarthritis was proven to be a paraneoplastic phenomenon of ALL.

A total of 1 week after the administration of chemotherapy (Idarubicin, $15 \mathrm{mg}$; cyclophosphamide, 1,200 mg; vindesine, $4 \mathrm{mg}$; once a week), the patient's arthritic symptoms were dramatically relieved without administration of any NSAIDs, which further suggested that sacroiliitis was an onset presentation of ALL in the present patient. During $>2$ years of follow-up, the patient experienced a complete remission and did not develop any lower back pain. Computed tomography scans of the sacroiliac joints were stable compared with those performed 2 years previously.

\section{Discussion}

Paraneoplastic syndromes are the manifestation of an underlying malignancy that may be hidden and may be caused by various tumor effects, including substances released by tumor cells, for example hormones, peptides and antibodies, or immunological and other host reactions to the tumor (3). The pathogenesis of paraneoplastic syndrome is complicated and largely remains to be elucidated. Manifestations of rheumatic disease, including arthritis, myositis, lupus-like syndrome and vasculitis, may be an initial presentation of malignancy in the form of paraneoplastic syndrome. Rheumatic symptoms may complicate diagnosis, but are significant indicators of an underlying malignancy.

Leukemic arthritis (LA) is a major form of paraneoplastic rheumatic syndrome, which may be defined as joint pain and

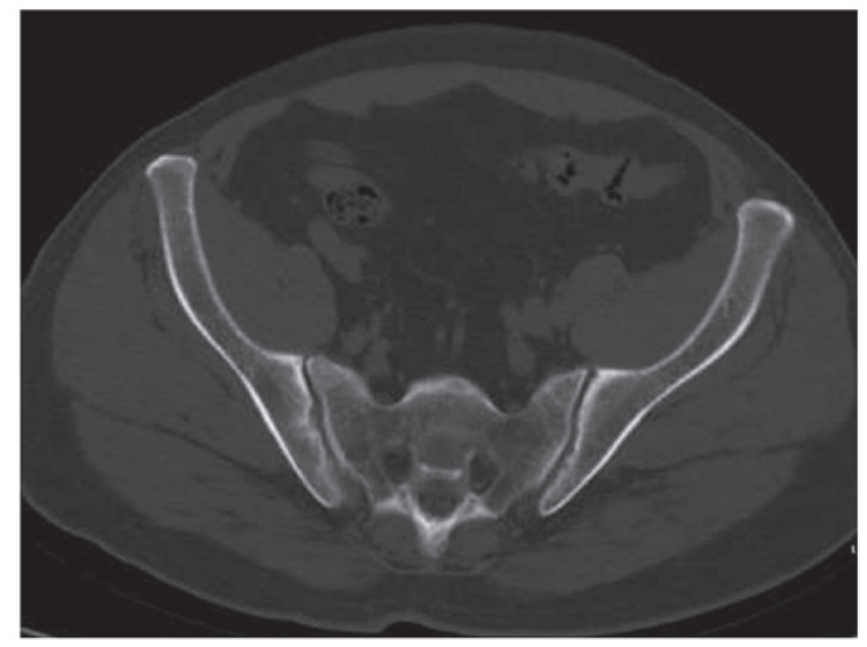

Figure 1. Computed tomography scan of the sacroiliac joints revealing bilateral erosion and sclerosis in the margin of the iliac side.

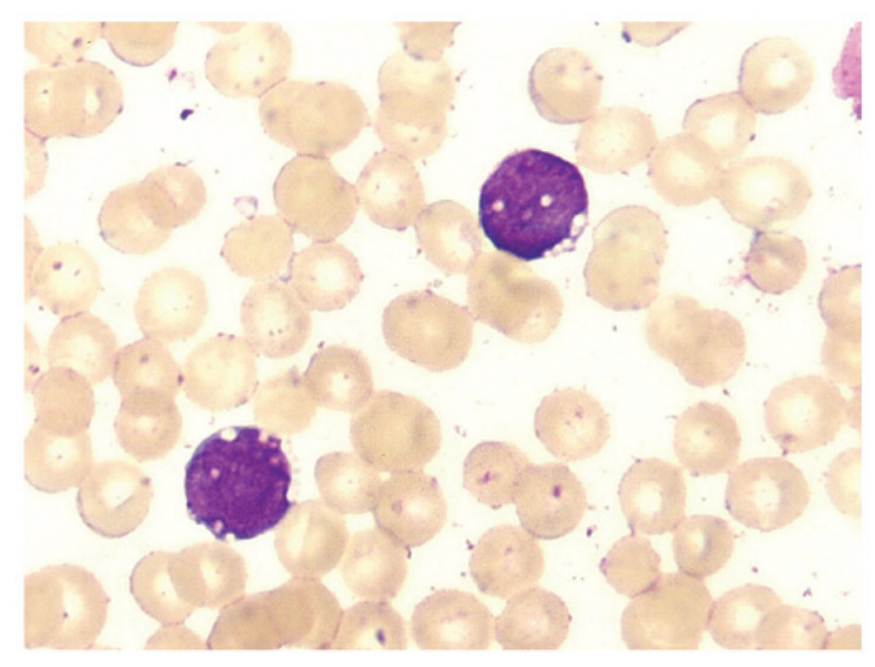

Figure 2. Primitive lymphocytes and prolymphocytes were identified in the bone marrow, and acute lymphocytic leukemia was indicated. Wright-Giemsa staining; magnification, x1,000.

swelling associated with peripheral blood or bone marrow leukemia, following the exclusion of alternative causes of arthritis (4). Potential pathogenic mechanisms for LA include hemorrhage into the joint due to thrombocytopenia, infiltration of leukemic cells into synovial tissue, immune complex-induced synovitis and synovial reaction to periosteal or capsular infiltration. However, synovial infiltration of leukemic cells appears to be the primary mechanism (5). Peripheral joints are frequently affected in LA mimicking rheumatoid arthritis, while axial joints, including the sacroiliac joint, are rarely affected $(6,7)$. Sacroiliitis as the onset symptom of malignancy is rare. To the best of our knowledge, the present patient is only the second case of ALL initially presenting with sacroiliitis reported in the relevant literature.

Leukemia presenting with arthritis has been reported in a small number of cases, while sacroiliitis at onset presentation is rare. In the previous literature, only two cases of leukemia initiated with sacroiliitis have been reported. In one case report, a 
28-year-old woman rapidly developed acute myeloid leukemia 1 month after the diagnosis of myelodysplastic syndrome with sacroiliitis (6). In another case report, a 17-year-old male patient was admitted to hospital due to experiencing a fever, lower back pain and knee pain for a number of months. A complete blood count revealed pancytopenia, and ALL was ultimately diagnosed (7). To the best of our knowledge, the present patient is the second case of ALL presenting with sacroiliitis reported in the relevant literature. Spondyloarthritis is a chronic inflammatory disease, primarily involving the axial spine and the sacroiliac joint (8). The condition typically occurs in young males and has a strong polygenic predisposition. Sacroiliitis tends to be the early manifestation of spondyloarthritis (8). The initial symptom is typically chronic lower back pain, which is often severe at rest, relieved by physical activity and accompanied by morning stiffness (8). In the current study, the patient presented with chronic lower back pain, and sacroiliitis was indicated by imaging. Furthermore, an elevated erythrocyte sedimentation rate and high C-reactive protein level were observed, mimicking spondyloarthritis. Subtle abnormalities in blood counts, an enlarged spleen and a poor response to NSAIDs were indicators of ALL. Clinicians should consider that spondyloarthritis may be the onset manifestation of ALL, although this is rare.

Infiltration, including into the bone, joints, liver, spleen or lymph nodes, is common in ALL, and ALL is prone to complications due to paraneoplastic rheumatic syndrome, particularly arthritic manifestations (9). As ALL typically affects children aged 3-7, and the majority of childhood leukemia cases are ALL, childhood LA is common while adult LA is rarely observed (1) Childhood LA is liable to be misdiagnosed as juvenile idiopathic arthritis (JIA). Marwaha et al (10) retrospectively analyzed the case records of 762 patients exhibiting ALL. The age of onset ranged from 1-14 years, and 49 patients $(6.4 \%)$ had an initial presentation that mimicked JIA. This subset of ALL patients may possess an improved prognosis and included an increased percentage of patients opting for therapy or those with a lower total leukocyte count, a higher platelet count and a reduced degree of anemia. Large joints of the limbs were most commonly affected, including the knee, ankle, hip, wrist, elbow and shoulder, while the spine and sacroiliac joint were not mentioned (10). Although peripheral joint involvement is common in LA, axial spinal involvement in leukemia should additionally be considered during the differential diagnosis.

LA is associated with monocytic leukemia in adults, potentially due to the significant role of monocytes in the pathogenic mechanism of arthritis. Acree et al (11) initially reported the association between adult LA and leukemia of monocytic differentiation. The study identified that four cases investigated in their study, and the majority of the prior reported cases of adult LA, were monocytic leukemia, indicating that adult LA occurs in association with leukemia of monocytic differentiation (11). Monocyte-attracting cytokines, including tumor necrosis factor $\alpha$, have a significant role in osteoarthritis and rheumatoid arthritis via mediation of and increasing monocyte migration into the inflamed joint space. Acree et al (11) hypothesized that leukemic monocytes, identical to their benign counterparts, may migrate to sites of inflamed joints in response to monocyte-attracting cytokines, which may be critical in the mechanism of LA. Although the present case was not monocytic leukemia, there were significantly increased levels of monocytes $\left(1.86 \times 10^{9} / 1 ; 36.3 \%\right)$. Increased monocyte levels may additionally have had a significant role in the mechanism of LA in the present case.

In the current relevant literature, clinical features, including night pains, alterations in blood counts, an absence of response to NSAID therapy and a fever, were considered to be suspected symptoms of ALL $(10,12,13)$. A low platelet count and night pain that wakes a child from sleep appear to be particularly sensitive indicators of ALL with arthritic symptoms $(12,13)$. For these patients, the examination of blood smears, bone scans and bone marrow examination should be conducted to exclude the possibility of ALL. In the present case report, a fever, a poor response to NSAID therapy, an enlarged spleen, anemia, a low platelet count and a low neutrophil count indicated the underlying hematological malignancy.

In conclusion, sacroiliitis may be a rare onset presentation of ALL. For patients exhibiting arthritic symptoms, particularly children and adolescents, atypical clinical features such as any subtle abnormalities in blood count and poor responses to therapy, indicate the requirement to exclude the possibility of acute leukemia. Early diagnosis is critical for treatment, and chemotherapy is frequently effective for treatment of the paraneoplastic rheumatic syndrome caused by ALL.

\section{References}

1. Usalan C, Ozarslan E, Zengin N, Büyükaýk Y and Güllü YH: Acute lymphoblastic leukaemia presenting with arthritis in an adult patient. Postgrad Med J 75: 425-427, 1999.

2. Jones OY, Spencer CH, Bowyer SL, Dent PB, Gottlieb BS and Rabinovich CE: A multicenter case control study on predictive factors distinguishing childhood leukemia from juvenile rheumatoid arthritis. Pediatrics 117: e840-e844, 2006.

3. Andras C, Csiki Z, Ponyi A, Illés A and Dankó K: Paraneoplastie rheumatic syndromes. Rheumatol Int 26: 376-382, 2006.

4. Luzar MJ and Sharma HM: Leukemia and arthritis: Including reports on light, immunofluorescent, and electron microscopy of the synovium. J Rheumatol 10: 132-135, 1983.

5. Taillan B, Leyge JF, Fuzibet JG, Nectoux F, Ziegler G and Dujardin P: Knee arthritis revealing acute leukemia in a patient with rheumatoid arthritis. Clin Rheumatol 10: 76-77, 1991.

6. Hoshino T, Matsushima T, Saitoh Y, Yamane A, Takizawa M, Irisawa H, Saitoh T, Handa H, Tsukamoto N, Karasawa M, et al: Sacroiliitis as an initial manifestation of acute myelogenous leukemia. Int J Hematol 84: 421-424, 2006.

7. Moghadam A, Talebi-Taher M and Dehghan A: Sacroiliitis as an initial presentation of acute lymphoblastic leukemia. Acta Clin Belg 65: 197-199, 2010.

8. Dougados M and Baeten D: Spondyloarthritis. Lancet 377: 2127-2137, 2011.

9. Appelbaum FR: The acute leukemias. Wyngaarden JB, Smith LH, Bennett JC (Eds). In: Cecil Textbook of Medicine. Volume 1 $19^{\text {th }}$ Edition. WB Saunders Company, Philadelphia, PA, USA, pp 44-949, 1992.

10. Marwaha RK, Kulkarni KP, Bansal D and Trehan A: Acute lymphoblastic leukemia masquerading as juvenile rheumatoid arthritis: Diagnostic pitfall and association with survival. Ann Hematol 89: 249-254, 2010

11. Acree SC, Pullarkat ST, Quismorio FP Jr, Mian SR and Brynes RK: Adult leukemic synovitis is associated with leukemia of monocytic differentiation. J Clin Rheumatol 17: 130-134, 2011.

12. Jones OY, Spencer CH, Bowyer SL, Dent PB, Gottlieb BS and Rabinovich CE: A multicenter case-control study on predictive factors distinguishing childhood leukemia from juvenile rheumatoid arthritis. Pediatrics 117: e840-e844, 2006.

13. Gupta D, Singh S, Suri D, Ahluwalia J, Das R and Varma N: Arthritic presentation of acute leukemia in children: Experience from a tertiary care centre in North India. Rheumatol Int 30: 767-770, 2010. 